\title{
Pure Dysarthria Resulting from a Small Cortical Infarction Located at the Left Middle Frontal Gyrus
}

\author{
Akiyuki Uzawa, Akiyuki Hiraga and Ikuo Kamitsukasa
}

\begin{abstract}
Pure dysarthria caused by a small cortical infarction is rare. Recent reports have revealed that a small cortical lesion can lead to pure dysarthria, furthermore some reports have revealed that a middle frontal gyrus lesion might cause pure dysarthria. We report a 64-year-old woman who presented only dysarthria and had a small limited cortical infarction located at the left middle frontal gyrus. This case indicated that an isolated middle frontal gyrus lesion can cause pure dysarthria by secondary compromising of the cortical areas related or connected to the corticobulbar tract.
\end{abstract}

Key words: pure dysarthria, middle frontal gyrus, cortical infarction, cerebral infarction, left hemisphere, stroke

(Inter Med 48: 75-76, 2009)

(DOI: 10.2169/internalmedicine.48.1540)

\section{Introduction}

Pure dysarthria was first described by Fisher as a variant of lacunar syndrome, caused by a pontine base infarction, without other neurological deficits (1). Following this report, some authors presented cases with unilateral lacunar infarction with minimal sensory or motor dysfunction (2-4). In the majority of cases, pure dysarthrias are caused by subcortical lesions, but in some cases cortical lesions cause pure dysarthria $(2,3,5-7)$. All extracerebellar infarctions were more frequent and more severe, when caused by left-sided lesions and were located along the course of cortico-bulbar tract fibers (8). Recent reports using diffusion-weighted imaging (DWI) have revealed that a small cortical lesion can lead to PD $(2,3)$.

\section{Case Report}

We present a woman who complained only of dysarthria and had a small cortical infarction located at the left middle frontal gyrus (MFG). A 64-year-old right-handed woman with a history of hyperlipidemia was admitted to our hospital because of sudden onset of dysarthria. On admission, she was alert and her blood pressure was $184 / 95 \mathrm{mmHg}$. Neuro- logical examination revealed mild dysarthria without non fluent aphasia, anarthria, or apraxia of speech. Facial paresis, oro-facial apraxia, dysphagia, or compromising tongue motility was not observed. The Medical Research Council grades of muscle power for all four limbs were 5/5. Tendon reflexes, sensory, and coordination examination were normal. Brain magnetic resonance imaging (MRI) taken 3 hours after onset using DWI and 18 hours after onset using T2weighted imaging (T2WI) showed a hyperintense lesion in the left MFG (Fig. 1). Brain MR angiography and electrocardiogram was normal. We considered that the ischemia was caused by small-vessel occlusion, and she underwent drip infusion of heparin and oral anti-platelet therapy. Her dysarthria improved gradually, and she was discharged without neurological deficit 6 days after onset.

\section{Discussion}

Most previous pure dysarthria cases resulting from small cortical lesions were located at the 'motor vocal area', thought to be in the precentral gyrus or corticobulbar tract, and the responsibile lesions were lateral to the precentral knob and at the most lateral cortical area in the level of the lateral ventricle, which probably involves the corticobulbar tract (3). Two cases of pure dysarthria by cortical infarction 


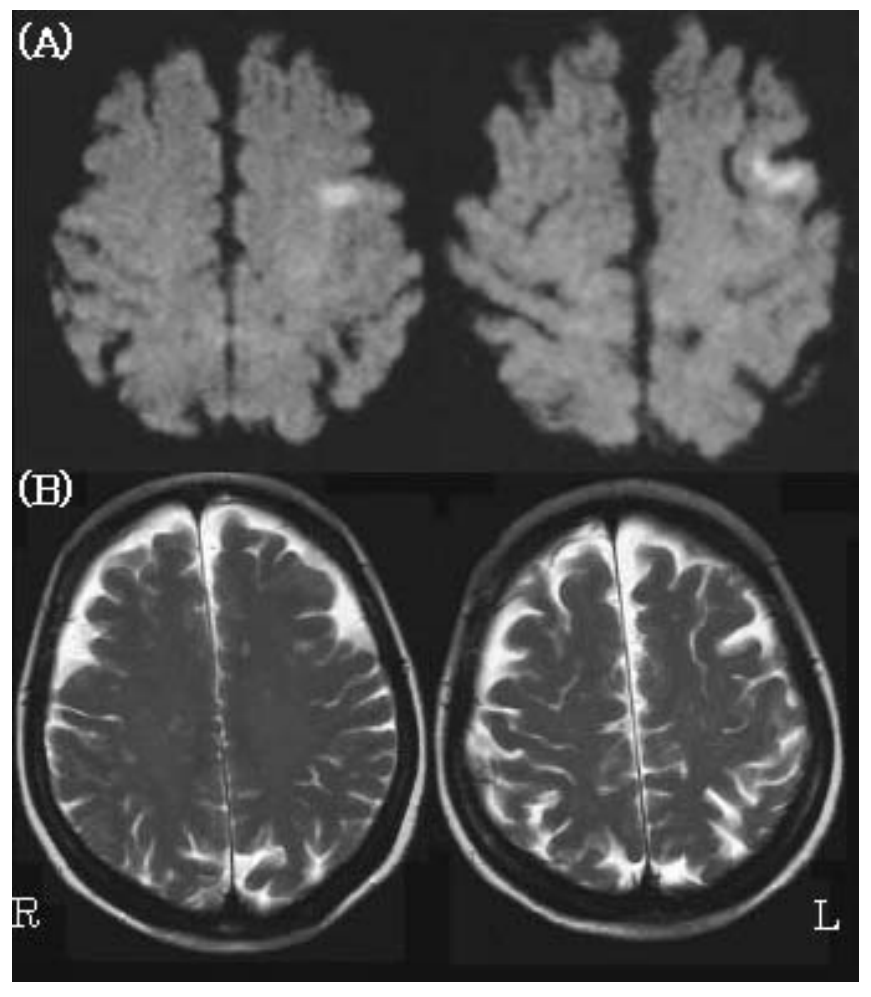

Figure 1. Diffusion-weighted imaging (DWI) taken 3 hours after onset (A) and T2-weighted imaging (T2WI) taken 18 hours after onset (B), showing a small high signal intensity lesion in the left middle frontal gyrus. involving a lesion of the MFG were reported (2). But those lesions were relatively extensive and involved both the MFG and the area lateral to the precentral knob. Therefore, the association of MFG with pure dysarthria in those cases was questionable. The present case however, had a small limited lesion in the left MFG without a precentral gyrus lesion and indicated that isolated MFG region can cause dysarthria, which could be secondary to compromise of the cortical areas related or connected to the corticobulbar tract. But it can not necessarily be denied that her dysarthria was caused by transient hypo-perfusion of other areas such as the precentral gyrus. On the other hand, dysarthria and facial paresis syndrome associated with cortical lacunar lesion, also involving MFG, has been described (8). The present case was also unique in that facial paresis was not observed. In addition, a previous report showed that dysarthria by cerebral infarction affected much more of the left hemisphere (9) as in our case. Although previous reports are unclear whether the MFG is associated with the speech motor function, this case and previous cases $(2,8)$ provide direct clinical evidence that a small infarction of the MFG can cause pure dysarthria.

\section{References}

1. Fisher CM. Lacunar strokes and infarcts: a review. Neurology 32: 871-876, 1982.

2. Gatto EM, Roca CU, Zurrú MC, Rugilo CA. Pure dysarthria due to small cortical stroke. Neurology 62: 345-346, 2004.

3. Kim JS, Kwon SU, Lee TG. Pure dysarthria due to small cortical stroke. Neurology 60: 1178-1180, 2003.

4. Tsugawa J, Tsuboi Y, Inoue H, Saitoh H, Yamada T. A small cortical infarction showing dysarthria and left hypoglossal paresis. Brain Nerve 59: 1373-1376, 2007.

5. Urban PP, Wicht S, Hopf HC, Fleischer S, Nickel O. Isolated dysarthria due to extracerebellar lacunar stroke: a central monoparesis of the tongue. J Neurol Neurosurg Psychiatry 66: 495-501,
1999.

6. Ichikawa K, Kageyama Y. Clinical anatomic study of pure dysarthria. Stroke 22: 809-812, 1991.

7. Kim JS. Pure dysarthria, isolated facial paresis, or dysarthria-facial paresis syndrome. Stroke 25: 1994-1998, 1994.

8. Urban PP, Wicht $S$, Vukurevic $G$, et al. Dysarthria in acute ischemic stroke: lesion topography, clinicoradiologic correlation, and etiology. Neurology 56: 1021-1027, 2001.

9. Urban PP, Rolke R, Wicht S, et al. Left-hemispheric dominance for articulation: a prospective study on acute ischaemic dysarthria at different localizations. Brain 129: 767-777, 2006.

(C) 2009 The Japanese Society of Internal Medicine http://www.naika.or.jp/imindex.html 